\title{
Puntaje LVIS como predictor de mortalidad en choque séptico
}

\author{
Humberto Alfonso Castillejos Suastegui, ${ }^{*}$ Enrique Monares Zepeda, ${ }^{*}$ Axel Pedraza Montenegro, \\ Alma Rosa Contreras Contreras, ${ }^{*}$ Careli Gómez Moctezuma, ${ }^{*}$ Janet Silvia Aguirre Sánchez, \\ Gilberto Camarena Alejo, ${ }^{*}$ Juvenal Franco Granillo*
}

\section{RESUMEN}

Introducción: El uso de vasopresores en choque séptico es parte fundamental del tratamiento. El uso de un puntaje que integre los múltiples vasopresores e inotrópicos como LVIS (levosimendan vasopressor inotropic score), puede ser de utilidad para el seguimiento y pronóstico de estos pacientes. Se ha estudiado en población pediátrica y no ha sido validada en población adulta.

Material y métodos: Estudio retrospectivo, longitudinal, retrolectivo en pacientes adultos admitidos a UCI con diagnóstico de choque séptico con el fin de investigar si el puntaje LVIS es capaz de predecir mortalidad y lesión renal aguda. Seguimiento a 30 días.

Resultados: Incluimos 77 pacientes, 24 (21.2\%) pacientes murieron y 41 $(53.23 \%)$ presentaron lesión renal aguda. Se observó un AUC (área bajo la curva) para LVIS 0.89 con un punto de corte de 21.3 (sensibilidad $50 \%$ y especificidad de $82 \%$ ). El puntaje LVIS > 21.3 con RR 2.09 (IC95\% 1.15-3.7, p $=0.003$ ) y un HR de 3.8 (IC95\% 1.5-9.3, $p=0.003), L R+2.81$ y LR- 0.61 para mortalidad. LVIS no fue significativo para predecir LRA (lesión renal aguda). Conclusiones: LVIS es útil para predecir mortalidad en choque séptico, con un punto de corte 21.3. Es necesario continuar estudios para validarlo en población adulta con otras formas de choque.

Palabras clave: Sepsis, choque séptico, mortalidad, vasopresores, inotrópicos.

\section{SUMMARY}

Introduction: Vasopressors have a fundamental roll in the treatment of septic shock. LVIS (levosimendan vasopressor inotropic score) as a score that integrates the main vasopressors and inotropic drugs may be useful for monitoring and prognosis. It's been studied in pediatric population, but not validated in adult patients so far.

Material and methods: Retrospective, longitudinal, retrolective study in adults admitted in ICU with septic shock. We looked to investigate if LVIS score is useful to predict mortality and acute kidney injury in ICU, with a follow-up of 30 days.

Results: We included 77 patients, 24 (21.2\%) patients died and 41 (53.23\%) developed acute kidney injury. LVIS had an AUC of 0.89 with a cut-off of 21.3 (50\% sensitivity and $82 \%$ of specificity). LVIS score above the cut-off 21.3 , had RR 2.09 (CI95\% 1.15-3.7, $p=0.003$ ), HR de 3.8 (Cl 1.5-9.3, $p=0.003$ ), LR+ 2.81 and LR- 0.61 for mortality. LVIS could not predict AKI (acute kidney).

Conclusions: LVIS score is useful to predict mortality in patients with septic shock, with a cut-off of 21.3. More research is left to be done to validate this score in other forms of shock in adult population.

Key words: Sepsis, septic shock, mortality, vasopressors, inotropic.

\section{RESUMO}

Introdução: O uso de vasopressores no choque séptico é uma parte fundamental do tratamento. O uso de um escore que integra múltiplos vasopressores e drogas inotrópicas como o LVIS (Levosimendan Vasopressor Inotropic Score), pode ser útil para o acompanhamento e prognóstico desses pacientes. Foi estudado na população pediátrica e não foi validado na população adulta.

Material e métodos: Estudo retrospectivo, longitudinal, retrolectivo em pacientes adultos admitidos na UTI com o diagnóstico de choque séptico para investigar se o escore LVIS é capaz de predizer a mortalidade e a lesão renal aguda em um acompanhamento de 30 dias.

Resultados: Foram incluídos 77 pacientes, 24 (21.2\%) pacientes morreram e 41 (53.23\%) apresentaram lesão renal aguda. Uma AUC (área sob a curva) foi observada para LVIS 0.89 com um ponto de corte de 21.3 (sensibilidade $50 \%$ e especificidade 82\%). O escore LVIS > $21.3 \mathrm{com} R R 2.09$ (IC95\% 1.15-3.7, $p=$ 0.003 ) e uma HR de 3.8 (IC95\% 1.5-9.3, $p=0.003$ ), $L R+2.81$ e $L R-0.61$ para mortalidade. O LVIS não foi significativa na predição da LRA (lesão renal aguda). Conclusões: O LVIS é útil para prever a mortalidade no choque séptico, com um ponto de corte de 21.3. É necessário continuar os estudos para validá-lo na população adulta com outras formas de choque.

Palavras-chave: Sepse, choque séptico, mortalidade, vasopressores inotrópicos.

* Centro Médico ABC.

Recepción: 01/09/2017. Aceptación: 06/09/2018.

Este artículo puede ser consultado en versión completa en http://www.medigraphic.com/medicinacritica

\section{INTRODUCCIÓN}

Actualmente se define a la sepsis como un estado de falla orgánica secundario a un proceso infeccioso; el choque séptico es un espectro de la enfermedad. ${ }^{1}$

Choque séptico se define como una anormalidad circulatoria y metabólica que incrementa la mortalidad y a nivel operativo se reconoce por la presencia de sepsis y el uso de vasopresores para mantener presión arterial media de $>65 \mathrm{mmHg}$ y la presencia de lactato $>2 \mathrm{mmol} / \mathrm{L}$ a pesar de la resucitación adecuada con líquidos. ${ }^{1}$

Los síndromes sépticos tienen una incidencia global de $437 / 100,000$ personas/año y representan $5.2 \%$ de los costos hospitalarios en Estados Unidos. ${ }^{2}$

El choque séptico es uno de los principales diagnósticos de ingreso a terapia intensiva, y está ligado a gran mortalidad en UCI (Unidad de Cuidados Intensivos), con hasta $37 \%$; requiere manejo multidisciplinario, y si bien el tratamiento del foco séptico más el uso de antibióticos son la base del tratamiento, también los fármacos vasopresores e inotrópicos y otros métodos de soporte vital son requeridos. ${ }^{3}$

Múltiples sociedades han concluido que el fármaco de primera elección es la norepinefrina. El uso de vasopresina se ha reconocido como benéfico, y la combinación de ambos ha dado resultados inconstantes. En un metaanálisis reciente, no sólo se confirma lo anterior sino que también se considera el uso de inodilatadores asociados, de los cuales el levosimendán se relaciona con menor mortalidad. La plausibilidad biológica de lo anterior estriba en la compleja interacción a nivel de la microvasculatura, lugar donde se realiza transporte de oxígeno, pudiendo mejorar el $\mathrm{VO}_{2}$ regional con mejoría clínica de los pacientes. ${ }^{4}$

El uso de vasopresores es fundamental en choque séptico ya que éstos permiten mantener la perfusión a nivel tisular y lograr una ventana de tiempo (junto al control del foco infeccioso) para revertir el proceso inflamatorio subyacente.

Intuitivamente, los clínicos asumen que el estado del paciente es más grave a medida que requiere dosis mayores de vasopresores o la combinación de varios fármacos. Sviri y colaboradores, en un estudio retrospectivo que incluyó 166 pacientes, dividieron a los pacientes con altas y bajas dosis de vasopresores; se definió arbitrariamente mayor o menor de $40 \mu \mathrm{g} / \mathrm{min}$ (norepinefrina o adrenalina). Se observó que aquellos pacientes que requirieron dosis altas de vasopresores presentaron una mortalidad de hasta $84.3 \%$, con 
un OR para mortalidad de 5.1 (IC 95\% de 2.02-12.9, p $=0.001)$. La dosis de $28 \mu \mathrm{g} / \mathrm{min}$ tuvo un AUC de 0.79 con una sensibilidad de $73.3 \%$ y especificidad de $75.4 \%$ para predecir mortalidad. Este trabajo demostró que a mayor requerimiento de vasopresores el paciente tiene peor pronóstico. ${ }^{5,6}$

Farkas y colaboradores reportaron los resultados de un estudio retrospectivo que incluyó a 1,023 pacientes con múltiples patologías admitidos a $\mathrm{UCl}$; se documentó que la supervivencia del grupo que requirió vasopresores fue de tan sólo $29.6 \%$, en comparación a $92 \%$ en el grupo que no ameritó vasopresores. ${ }^{7}$

Aunque a mayor requerimiento de vasopresores la mortalidad se incrementa, hay que aclarar que estos fármacos por sí mismos no están asociados a deterioro en los pacientes (aunque pueden llegar a tener efectos colaterales) y no tienen un impacto por sí mismos en la mortalidad. El incremento de la mortalidad a mayor dosis de vasopresores es reflejo de la carga inflamatoria subyacente. $^{8}$

Específicamente, en pacientes con choque séptico también se ha establecido relación lineal entre el incremento en la dosis de vasopresores y la mortalidad. En una cohorte de 106 pacientes, se ajustó la dosis de vasopresor con base en el promedio del peso (WMD por sus siglas en inglés), observándose que una dosis $>0.75 \mu \mathrm{g} / \mathrm{kg} / \mathrm{min}$ estaba asociada a mortalidad con sensibilidad de $73 \%$ y especificidad de $74 \%$. Si además se agregaba SOFA > 10 puntos la mortalidad llegaba a $87 \% .^{9}$

Desde el 2013 se consideró normalizar con base en equivalencias de norepinefrina otros fármacos vasopresores. Brown y colaboradores establecieron una equivalencia para las dosis de norepinefrina y otros fármacos vasopresores; observaron que aquellos pacientes con un equivalente $>1 \mu \mathrm{g} / \mathrm{kg} / \mathrm{min}$ de norepinefrina sobrevivieron $17 \%$ a 90 días. Cincuenta y tres por ciento de la población de dicho estudio eran pacientes con choque séptico. ${ }^{10}$

Existe relación directa entre la dosis de vasopresores empleada y la mortalidad. No obstante, en los estudios realizados en adultos no se toma en consideración el uso de inotrópicos en conjunto con vasopresores, y es difícil considerar la contribución de cada fármaco si se toma por separado. Por lo tanto, normalizar las diferentes dosis e integrarlas en un solo puntaje es útil en el pronóstico del paciente hemodinámicamene comprometido.

Davidson y colaboradores emplearon el puntaje de vasoactivos e inotrópicos (VIS) en población pediátrica posterior a cirugía cardiaca:

VIS = Dopamina $(\mu \mathrm{g} / \mathrm{kg} / \mathrm{min})+$ Dobutamina $(\mu \mathrm{g} / \mathrm{kg} /$ min $)+100 \times$ epinefrina $(\mu \mathrm{g} / \mathrm{kg} / \mathrm{min})+10 \times$ milrinona $(\mu \mathrm{g} / \mathrm{kg} / \mathrm{min})+10,000+$ vasopresina $(U \mathrm{l} / \mathrm{kg} / \mathrm{min})+100 \times$ norepinefrina $(\mu \mathrm{g} / \mathrm{kg} / \mathrm{min}) .{ }^{11}$
En dicho estudio se observó mayor tiempo de estancia, ventilación mecánica y presencia de falla renal con puntaje VIS elevado. Se observó que en esta población el puntaje más relevante fue el que se obtuvo a las 48 horas con un punto de corte de 10.2 y un AUC de 0.93 . Cabe señalar que no se documentó ninguna muerte en ese estudio. ${ }^{11}$

Favia y colaboradores sugirieron agregar levosimendán al VIS considerando el impacto de dicho inodilatador en la población postoperada de cirugía cardiovascular teniendo como fórmula final:

LVIS = Dopamina $(\mu \mathrm{g} / \mathrm{kg} / \mathrm{min})+$ Dobutamina $(\mu \mathrm{g} / \mathrm{kg} /$ min) $+100 \times$ epinefrina $(\mu \mathrm{g} / \mathrm{kg} / \mathrm{min})+10 \times$ milrinona $(\mu \mathrm{g} / \mathrm{kg} / \mathrm{min})+10,000+$ vasopresina $(\mathrm{Ul} / \mathrm{kg} / \mathrm{min})+100$ $\times$ norepinefrina $(\mu \mathrm{g} / \mathrm{kg} / \mathrm{min})+50 \times$ levosimendán $(\mu \mathrm{g} /$ $\mathrm{kg} / \mathrm{min})^{12}$

El uso del puntaje de vasopresores e inotrópicos ha sido explorado recientemente en pacientes con choque séptico; también en población pediátrica. Cabe señalar que el artículo no establece un punto de corte para VIS, pero se concluye que el cálculo de un puntaje a las 48 horas puede predecir tiempo de estancia así como riesgo para intubación. Por otro lado, el cálculo de VIS entre las 12-24 horas se relacionó con riesgo de paro cardiaco, uso de ECMO o muerte. ${ }^{13}$

\section{MATERIAL Y MÉTODOS}

Estudio de cohorte, observacional, longitudinal, retrospectivo, retrolectivo, que se realizó en dos unidades de cuidados intensivos de tercer nivel en la Ciudad de México, de enero de 2011 a marzo de 2015. El presente estudio se realizó como un subanálisis del trabajo de tesis Índice $\mathrm{VO}_{2} \mathrm{Max} / \mathrm{LVIS}$ como pronóstico en choque séptico (también realizado por los autores). Se calculó muestra para regresión lineal múltiple, con un mínimo de $\mathrm{n}=58$ para un poder meta de 0.9 y una $p<0.05$. El estudio tuvo como propósito demostrar utilidad del puntaje LVIS para predecir mortalidad en pacientes con choque séptico. En la Figura 1 se esquematiza el diseño del estudio.

Criterios de inclusión: Sexo masculino y femenino, adultos mayores de 18 años, con diagnóstico de choque séptico de cualquier origen. Datos completos para el análisis de las variables. Pacientes ingresados en la Unidad de Terapia Intensiva o intermedia.

Criterios de no inclusión: Pacientes que provengan de otras instituciones con diagnóstico de choque séptico; pacientes con datos incompletos para el estudio; pacientes que hayan muerto en las primeras horas de su ingreso a terapia intensiva.

Variables del estudio: Se registró edad, sexo, talla, peso y se derivó el índice de masa corporal, presión sistólica al ingreso a terapia intensiva, SOFA 
(24 horas), SAPS III (al ingreso a terapia intensiva), creatinina al ingreso y se determinó con base en el registro de creatinina previa, lesión renal aguda (según los criterios de AKIN) y qué pacientes recibieron hemodiálisis. Se documentó saturación venosa central al ingreso, lactato al ingreso, procalcitonina al ingreso y a las 24 horas. Los tipos de vasopresores e inotrópicos fueron registrados así como las dosis empleadas en $\mu \mathrm{g} / \mathrm{min}$ y $\mu \mathrm{g} / \mathrm{kg} / \mathrm{min}$ según el caso, y el balance hídrico a las 72 horas. El seguimiento fue a 30 días y se documentaron los pacientes que murieron durante su internamiento.

Descripción general del estudio: Se incluyeron pacientes a través de muestra no aleatoria que cumplieran con los criterios de inclusión. No se realizó maniobra de intervención y se recabaron únicamente los datos clínicos y paraclínicos consignados en los expedientes, tomando datos de forma retrospectiva.

El diagnóstico de choque séptico se tomó con base en el diagnóstico de ingreso a terapia intensiva en aquellos pacientes con requerimiento de soporte vasopresor, sin importar el foco infeccioso o el agente etiológico; se recabó la información de estancia hospitalaria hasta por 30 días o hasta alcanzar el objetivo primario (muerte) o los objetivos secundarios (lesión renal aguda).

Cálculo de LVIS: Se calculó de forma manual el puntaje de vasopresores e inotrópicos (y levosimendán en los casos donde se aplique) a las 24 horas del ingreso a terapia intensiva. Los cálculos se hicieron con base en la fórmula propuesta por Favia y colaboradores. ${ }^{12}$

Fórmula 1. LVIS = Dopamina $\mu \mathrm{g} / \mathrm{kg} / \mathrm{min})+$ dobutamina $(\mu \mathrm{g} / \mathrm{kg} / \mathrm{min})+100 \times$ epinefrina $(\mu \mathrm{g} / \mathrm{kg} / \mathrm{min})+10 \times$ milrinona $(\mu \mathrm{g} / \mathrm{kg} / \mathrm{min})+10,000 \times$ vasopresina $(\mathrm{Ul} / \mathrm{kg} / \mathrm{min})+$ $100 \times$ norepinefrina $(\mu \mathrm{g} / \mathrm{kg} / \mathrm{min})+$ levosimendán $50 \times$ ( $\mu \mathrm{g} / \mathrm{kg} / \mathrm{min})$.

\section{Análisis estadístico}

Se utilizó estadística descriptiva para las variables numéricas que incluyeron medidas de tendencia central y dispersión, media o mediana y desviación estándar o rangos intercuartilares (RIQ). Las variables categóricas fueron representadas como frecuencias absolutas, relativas y probabilidades de riesgo con intervalos de confianza de $95 \%$. Las pruebas de hipótesis para variables lineales fueron analizadas con t de Student y Chi cuadrada para las variables categóricas. El puntaje de LVIS se incluyó en la construcción de modelos de regresión logística binaria con la mortalidad como variable dependiente. La fuerza de asociación expresada mediante Riesgo relativo (RR) o Hazard Ratios (HR) para los modelos de supervivencia e intervalos de confianza de $95 \%$ en ambos casos. Las variables lineales (SAPS III) fueron incluidas en modelos de regresión lineal multivariada y regresión de Cox para análisis de supervivencia. El rendimiento de las variables generadas y puntajes analizados para discriminar mortalidad fueron estudiados con el cálculo de la sensibilidad, especificidad, razones de verosimilitud positiva (RVP) y negativa (RVN) y la construcción de curvas de características operativas del receptor $(\mathrm{ROC})$ y las comparaciones de sus áreas bajo la curva (AUC), así como la identificación de puntos de corte con mayor rendimiento.

Todas las pruebas de hipótesis tendrán un error alfa ajustado para una $p<0.05$. La paquetería estadística empleada fue STATASE 11.0.

\section{Aspectos éticos}

Los procedimientos propuestos en la metodología de este estudio se apegaron a los lineamientos éticos contemplados para la investigación en seres humanos de la declaración de Helsinki de 1964, revisadas en el año 2000 [Asamblea Médica Mundial, 2000; Ley General de Salud, 1987].

De acuerdo a la Ley General de Salud en Materia de Investigación, se considera un estudio sin riesgo. El Comité de Ética de la institución aprobó el protocolo. Los investigadores no hicieron ninguna maniobra experimental. Se guardó la confidencialidad de todos los participantes.

\section{Recursos para el estudio, financiamiento y factibilidad}

No fue requerido financiamiento externo. En nuestra institución contamos con todos los insumos necesarios para realizar el estudio.

\section{RESULTADOS}

Incluimos un total de 77 pacientes, con una media de edad de 68 años (DE \pm 15 años), de los cuales $50.6 \%(n=39)$ fueron hombres y 38 mujeres $(49.4 \%)$; la media de índice de masa corporal fue de 26.38 (DE $\pm 6.69)$; la saturación venosa central al ingreso de $72 \%$ en promedio ( $D E \pm 11$ ); el promedio de lactato al ingreso fue de 2.64 (DE \pm 2.29 ); la media de procalcitonina al ingreso fue de 20.59 (DE \pm 50.47 ); se observó descenso a las 24 horas de ingreso, con una media de 11.05 (DE \pm 21.39 ); la media de balance hídrico a las 72 horas fue de 2,430 mL (DE \pm 4,676 $\mathrm{mL}$ ); la puntuación SAPS III media fue de 64 (DE \pm 13) y SOFA score 7.3 (DE \pm 3.5 ); la puntuación LVIS fue de 24.84 como media (Tablas 1 a 3) para el resto de los resultados.

De la población total, 41 (53.23\%) presentaron lesión renal aguda y 15 pacientes (19.5\%) requirieron hemodiálisis; Ia estancia media en UCI fue de 10 
Tabla 1: Características generales $(n=77)$.

\begin{tabular}{|c|c|c|}
\hline & Media & DE \\
\hline Edad & 68.000 & 15.000 \\
\hline \multicolumn{3}{|l|}{ Sexo } \\
\hline Masculino & 39.000 & $50.600 \%$ \\
\hline Femenino & 38.000 & $49.400 \%$ \\
\hline $\mathrm{IMC}\left(\mathrm{kg} / \mathrm{m}^{2}\right)$ & 26.380 & 6.690 \\
\hline $\mathrm{SvcO}_{2}$ al ingreso (\%) & 72.000 & 11.000 \\
\hline Lactato al ingreso (mmol/L) & 2.640 & 2.290 \\
\hline PCT al ingreso (ng/dL) & 20.590 & 50.470 \\
\hline PCT a las 24 horas (ng/dL) & 11.050 & 21.390 \\
\hline FC Máx. 24 horas (LPM) & 105.000 & 20.000 \\
\hline \multicolumn{3}{|l|}{ TFG } \\
\hline Dosis de norepinefrina ( $\mu \mathrm{g} / \mathrm{min})$ & 14.000 & 13.000 \\
\hline Dosis de vasopresina (Ul/min) & 0.034 & 0.029 \\
\hline Dosis dobutamina ( $\mu \mathrm{g} / \mathrm{kg} / \mathrm{min})$ & 5.000 & 20.000 \\
\hline Dosis levosimendán $(\mu \mathrm{g} / \mathrm{kg} / \mathrm{min})$ & 0.160 & 0.050 \\
\hline Días UCl & 10.000 & 12.000 \\
\hline Mortalidad 30 días & 24.000 & $31.200 \%$ \\
\hline Creatinina al ingreso (mg/dL) & 1.700 & 1.430 \\
\hline Lesión renal aguda & 41.000 & $53.230 \%$ \\
\hline \multicolumn{3}{|l|}{ Hemodiálisis } \\
\hline No & 62.000 & $80.500 \%$ \\
\hline Sí & 15.000 & $19.500 \%$ \\
\hline Balance a las 24 horas (mL) & $2,277.000$ & $2,546.000$ \\
\hline PAS al ingreso $(\mathrm{mmHg})$ & 109.000 & 23.000 \\
\hline SAPS III & 64.000 & 13.000 \\
\hline SOFA & 7.300 & 3.500 \\
\hline LVIS & 24.840 & 23.550 \\
\hline
\end{tabular}

Nota: Las variables demográficas se presentan como sus totales y porcentajes, las variables continuas y puntajes se presentan como medias y desviación estándar. $I M C=$ Índice de masa corporal, $\mathrm{SvCO}_{2}=$ Saturación venosa central, $\mathrm{PCT}=$ Procalcitonina, SAPS = Simplified Acute Physiologic Score, SOFA = Sequential Organ Failure Assessment. TFG = Tasa de filtrado glomerular. FC Máx. 24 horas = Frecuencia cardiaca máxima a las 24 horas. LVIS = Levosimendan vasopresores inotropic score. PAS = Presión arterial sistólica.
(DE \pm 12 ), finalmente, se documentaron 24 muertes (31.2\%). En la Tabla 2 se expresa el análisis univariado para mortalidad.

\section{Riesgo de mortalidad y análisis de supervivencia}

El análisis bivariado encontró que los puntos de corte identificados se asocian a mayor mortalidad para LVIS $\geq 21.3 \mathrm{RR}=2.09$ (IC95\% 1.15-3.7, $p=0.03)$. El tiempo total de exposición con una mediana de supervivencia fue de 27 días (RIQ 7-32) con un tiempo total de exposición de 790 días.

- LVIS $\geq 21.3$ con un HR = 3.8 (IC95\% 1.5-9.3, $p=0.003)$.

Con dicho punto de corte se obtuvo una sensibilidad de $50 \%$ y una especificidad de $82 \%$ con un valor predictivo positivo (VPP) $66 \%$ y un valor predictivo negativo de $69 \%$. La razón de verosimilitud (LR) positiva fue de 2.81 y LR negativa de 0.61 , con un área bajo la curva del operador de $0.82(0.71-0.92)$. En la Figura 2

\section{Tabla 3: Tipo de vasopresores e inotrópicos y distribución dentro de la población $(n=77)$.}

\begin{tabular}{lcc}
\hline Inotrópico o vasopresor & Número & Porcentaje \\
\hline Norepinefrina & 76 & 98.7 \\
Vasopresina & 45 & 58.4 \\
Dobutamina & 67 & 87.0 \\
Levosimendán & 7 & 9.1 \\
\hline
\end{tabular}

Tabla 2: Análisis de variables según mortalidad.

\begin{tabular}{|c|c|c|c|c|c|}
\hline & \multicolumn{2}{|c|}{$\begin{array}{l}\text { Supervivientes } \\
\qquad(n=53)\end{array}$} & \multicolumn{2}{|c|}{$\begin{array}{l}\text { Defunciones } \\
\qquad(n=24)\end{array}$} & \multirow[b]{2}{*}{$\mathrm{P}$} \\
\hline & Media & $\mathrm{DE}$ & Media & DE & \\
\hline Edad & 67.00 & 17.00 & 68.00 & 10.00 & 0.851 \\
\hline Masculino & 25.00 & $47.20 \%$ & 14.00 & $58.30 \%$ & 0.820 \\
\hline Femenino & 28.00 & $52.80 \%$ & 10.00 & $41.70 \%$ & \\
\hline IMC $\left(\mathrm{kg} / \mathrm{m}^{2}\right)$ & 26.99 & 6.89 & 25.05 & 6.16 & 0.242 \\
\hline $\mathrm{TFG}(\mathrm{mL} / \mathrm{min})$ & 60.00 & 26.00 & 57.00 & 29.00 & 0.699 \\
\hline FC máx. 24 horas (LPM) & 100.00 & 21.00 & 116.00 & 16.00 & 0.002 \\
\hline Presión arterial sistólica ingreso $(\mathrm{mmHg})$ & 110.00 & 22.00 & 107.00 & 26.00 & 0.632 \\
\hline $\mathrm{SvcO}_{2}$ ingreso (\%) & 72.00 & 11.00 & 73.00 & 12.00 & 0.742 \\
\hline Lactato ingreso (mmol/L) & 1.87 & 1.07 & 4.33 & 3.22 & $<0.001$ \\
\hline PCT ing $(\mathrm{ng} / \mathrm{mL})$ & 16.83 & 46.58 & 29.09 & 58.55 & 0.336 \\
\hline PCT 24 horas (ng/mL) & 9.45 & 15.64 & 15.44 & 32.70 & 0.342 \\
\hline Días UCl & 12.00 & 14.00 & 7.00 & 8.00 & 0.124 \\
\hline Hemodiálisis & 4.00 & 7.50 & 11.00 & 45.80 & $<0.001$ \\
\hline Balance 24 horas & $1,955.00$ & $2,151.00$ & $2,987.00$ & $3,194.00$ & 0.158 \\
\hline SAPS III & 61.00 & 12.00 & 70.00 & 13.00 & 0.004 \\
\hline SOFA & 6.00 & 3.00 & 10.00 & 4.00 & 0.001 \\
\hline LVIS & 15.87 & 10.50 & 44.66 & 31.44 & $<0.001$ \\
\hline
\end{tabular}

$\mathrm{DE}=$ Desviación estándar, IMC = Índice de masa corporal, FC = Frecuencia cardiaca, $\mathrm{SvcO}_{2}$ ing = Saturación venosa central ingreso, PCT = Procalcitonina, SAPS = Simplified acute physiologic score, SOFA = Sequential Organ Failure Assessment. LVIS = Levosimendan vassopresors inotropic score. LPM $=$ Latidos por minuto. TFG = Tasa de filtrado glomerular. 
se muestra la curva de supervivencia con el punto de corte establecido.

\section{Lesión renal aguda y mortalidad}

La incidencia de LRA fue de 53\%; adicionalmente, la mayor proporción de pacientes con LRA tuvieron desenlaces fatales; sin embargo, sin alcanzar diferencias estadísticamente significativas. $R R=1.02$ (IC 95\% $0.65-1.6, p=0.9$ ).

La escala propuesta demostró capacidad diagnóstica moderada para LRA.

- LVIS con AUC = 0.65 [IC95\% 0.52-0.77, $p=0.024)$.

Los pacientes con LRA, adicionalmente, mostraron puntajes más altos de LVIS 18.27 ( $D E \pm 18.53$ ) versus $30.61(\mathrm{DE} \pm 26.07) \mathrm{p}=0.021$.

\section{DISCUSIÓN}

El choque séptico es uno de los principales diagnósticos de ingreso a terapia intensiva, y está ligado a mortalidad de hasta $37 \%$. El manejo es multidisciplinario y el uso de antibióticos, así como el control del foco infeccioso, son la base del tratamiento. El uso de fármacos vasopresores e inotrópicos y otros métodos de soporte vital son requeridos.

La mortalidad registrada en el presente estudio fue de $31 \%$ conforme a lo reportado en la literatura mundial; la mayoría de los pacientes requirieron al me- nos dos vasopresores (norepinefrina y vasopresina); el inotrópico más empleado fue dobutamina y sólo a siete pacientes se les administró levosimendán. Se decidió emplear LVIS ya que incluye al levosimendán para el cálculo final del puntaje. La relevancia estriba en que en estudios recientes se ha reportado reducción de la mortalidad en pacientes con choque séptico y uso de levosimendán, aunque en el estudio LeoPARDS no se logró demostrar un impacto benéfico de este inodilatador en el contexto de choque séptico. ${ }^{14}$ Consideramos que la validación de este puntaje puede hacerse extensiva a otras formas de choque y abrir así una ventana para la investigación del mismo.

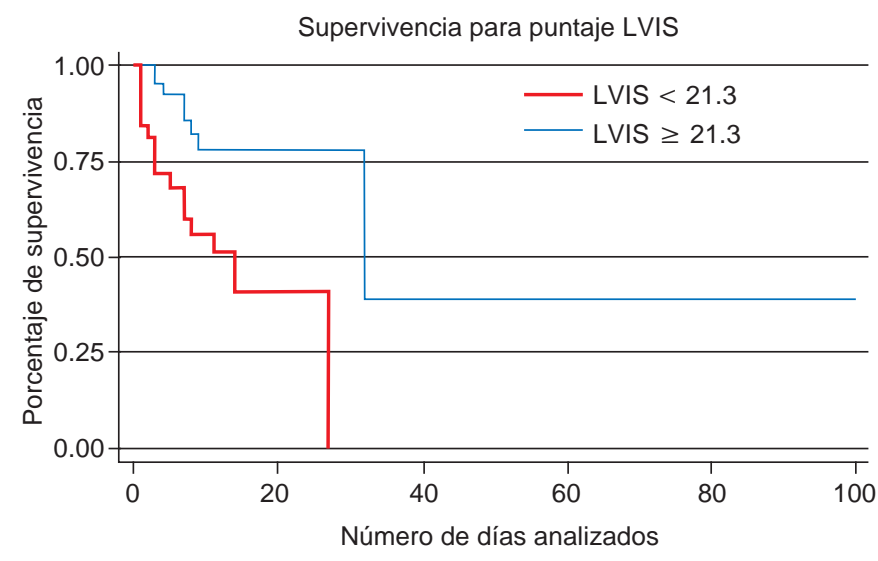

Figura 2: Curva de supervivencia para levosimendan vasopressor inotropic score.

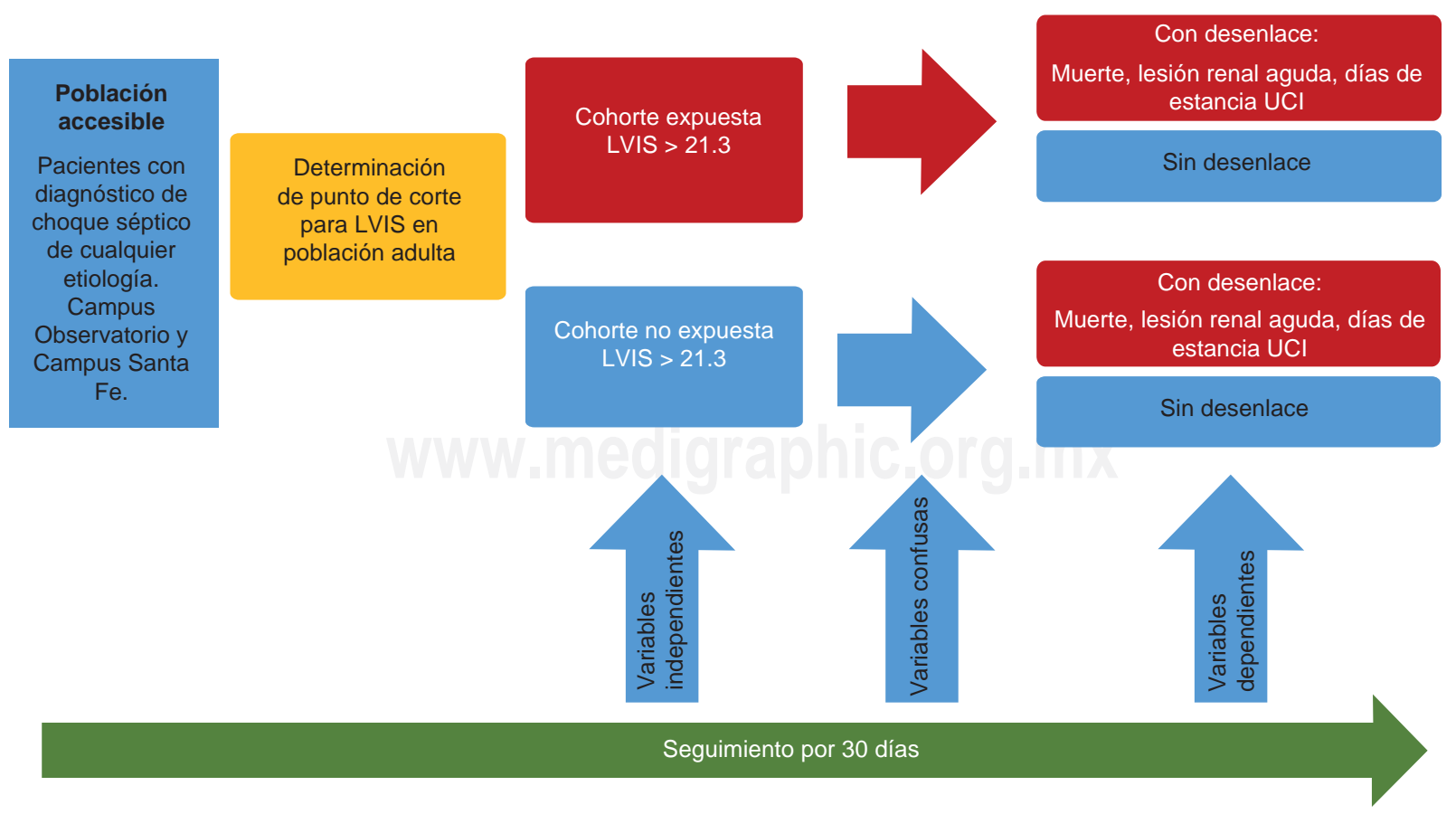

Figura 1: Esquema de diseño del estudio. 
Es de notar que la lesión renal aguda (empleando la definición actual del grupo AKIN), no fue un factor relacionado a mortalidad en el análisis univariado, probablemente en relación al número de pacientes que cumplieron con la definición: 41 (53.23\%), es decir, los criterios del grupo AKIN son muy sensibles, por lo cual creemos que una cohorte mayor podría demostrar el impacto de la LRA sobre la mortalidad. Cabe señalar que los pacientes que requirieron hemodiálisis presentaron mayor mortalidad, aunque no se investigaron los criterios de inicio de hemodiálisis ni el tiempo en que se inició la terapia.

En cuanto a los puntajes pronósticos, la puntuación SAPS III y SOFA están relacionados a mortalidad en el análisis univariado. Son puntuaciones altamente validadas, apegándonos a lo reportado en la literatura mundial.

En nuestro estudio se estableció la eficiencia de LVIS como factor pronóstico con una AUC de 0.82 y se determinó un punto de corte de 21.3, el cual tuvo la mejor relación entre sensibilidad y especificidad (50 y $82 \%$ respectivamente), con un HR de 3.8 (IC95\%1.59.3, $p=0.003$ ).

En el estudio realizado por Davidson y colaboradores, el punto de corte para el puntaje VIS fue de 10.5 con un AUC de 0.93 (IC de 95\% 0.85-1.00). Sin embargo, en dicho estudio la población era pediátrica, postoperados de cirugía cardiaca, y los objetivos principales fueron tiempo prolongado de intubación (OR 22.3 IC 95\% 3.1-157.7, $p=0.002$ ), tiempo prolongado de estancia en la UCI (OR 8.1 IC 95\% 1.4-45.4, p = $0.017)$ y tiempo prolongado de estancia en el hospital (OR 11.3 IC 95\% 1.7-73.7 p = 0.011). No se reportó mortalidad. ${ }^{11}$

Otra diferencia fundamental fue la temporalidad, ya que en dicho estudio la medición de VIS fue a las 48 horas de ingreso. Nosotros decidimos realizar la medición del puntaje LVIS a las 24 horas. Para esto nos basamos en el estudio realizado por McIntosh y colaboradores en pacientes pediátricos sépticos, en el cual se calculó el puntaje VIS, y se observó que para la combinación paro cardiaco, ECMO y muerte la medición a las 12-24 horas fue más eficiente como pronóstico, con un incremento en $14 \%$ de probabilidad por cada punto de incremento en VIS; sin embargo, no se reporta un punto de corte. ${ }^{13}$

El puntaje tiene utilidad moderada para predecir lesión renal aguda con un AUC de 0.65; sin embargo, ante puntajes más altos $(>30)$ dicho desenlace se presentó con mayor frecuencia $(\mathrm{p}=0.021)$ respecto a puntajes menores $(<19)$.

El presente trabajo es el primero que estudió el puntaje LVIS en población adulta, así como su capacidad para predecir mortalidad en contexto de choque séptico. Esto es relevante, puesto que con base en la experiencia de los clínicos y de forma intuitiva siempre se ha supuesto mayor gravedad del paciente crítico a mayor soporte vasopresor requerido. Hasta el momento no se ha logrado consensar y homogeneizar el concepto de dosis altas o bajas de vasopresores y menos integrar las dosis de cada fármaco vasopresor o inotrópicos como un puntaje de soporte hemodinámico, por lo que el uso de LVIS puede establecer con base en la mortalidad el concepto de dosis altas o bajas de soporte hemodinámico y continuar la monitorización del paciente.

El presente estudio tiene múltiples limitantes: en primer lugar, la fórmula LVIS fue tomada de estudios en población pediátrica, con el problema que suponen las equivalencias de fármacos y su integración en un solo puntaje, que pueden no ser válidas en adultos. El diseño del estudio también limita el peso de los resultados. Otra limitante es que en el estudio se incluyeron relativamente pocos pacientes con levosimendán, por lo que no se sabe el comportamiento de LVIS; se hace una comparación entre grupos con y sin levosimendán y se valoran las diferencias. Aunque el número de muestra fue alcanzado, creemos que debe incluir más pacientes, a fin de demostrar el rendimiento de LVIS como predictor de falla renal u otras fallas orgánicas, así como incluir variables hemodinámicas, respiratorias y ver la influencia de la etiología del choque séptico en la mortalidad y ajustarla a LVIS.

\section{CONCLUSIONES}

El puntaje LVIS en población adulta con choque séptico es capaz de predecir mortalidad, con un punto de corte de 21.3; sin embargo no fue concluyente para predecir lesión aguda. LVIS es una fórmula sencilla que permite expresar de forma conjunta la dosis de vasopresores e inotrópicos empleados en los pacientes, con implicaciones pronósticas relevantes. La fórmula requiere mayor validación en población adulta así como su desempeño en otras formas de choque.

\section{Agradecimientos}

Agradecemos a las autoridades de la institución así como a los médicos adscritos de las Unidades de Terapia Intensiva participantes.

\section{BIBLIOGRAFÍA}

1. Singer M, Deutschman CS, Seymur CW, Shankar-Hari M, et al. The third international consensus definitions for sepsis and septic shock. JAMA. 2016;315(8):801-810.

2. Fleischman C, Scherag A, Adhikari NK, Harlog CS, Tsaganos $\mathrm{T}$, et al. Assesment of global incidence and mortality of hospitaltreated sepsis. Current estimate and limitations. Am J Resp Crit Care Med. 2016;193(3):259-272.

3. Daviaud F, Grimaldi D, Dechartres A, Charpentier J, et al Timing and causes of death in septic shock. Ann Intensive Care. 2015;5:16. 
4. Beck V, Chateau D, Bryson GL, Pisipati A, et al. Timing of vasopressor initiation and mortality in septic shock: a cohort study. Crit Care. 2014;18(3):R97.

5. Belleti A, Benedetto U, Biondi-Zoccai G, Leggieri C, et al. The effect of vasoactive drugs on mortality in patients with severe sepsis and septic shock. A network meta-analysis of randomized trials. J Crit Care. 2017;37:91-88.

6. Sviri S, Hashoul J, Stav I, van Heerden PV. Does high-dose vasopressor therapy in medical intensive care patients indicate what we already suspect? J Crit Care. 2014;29:157-160.

7. Farkas DT, Rahnemai-Azar AA, Kunhammed SS, Greenbaum A, et al. Realistic survival outcomes after vasopressor use in the intensive care unit. Am J Hosp Palliat Care. 2016;33:871-874.

8. Belletti A, Castro ML, Silvetti S, Greco T, et al. The effect of inotropes and vasopressors on mortality: a meta-analysis of randomized clinical trials. Br J Anaesth. 2015;115:656-675.

9. Auchet T, Regnier MA, Girend N, Levy B. Outcome of patients with septic shock and high-dose vasopressor therapy. Ann Intensive Care. 2017:7:43.

10. Brown SM, Lanspa MJ, Jones JP, Kuttler KG, et al. Survival after shock requiring high-dose vasopressor therapy. Chest. 2013;143:664-671.

11. Davidson J, Tong S, Hancock $\mathrm{H}$, Hauck A, et al. Prospective validation of the vasoactive inotropic score and correlation to short term outcomes in neonates and infants after cardiothoracic surgery. Intensive Care Med. 2012;38:1184-1190.
12. Favia I, Vitale V, Ricci Z. The vasoactive -inotropic score and levosimendan: time for LVIS? J Cardiothorac Vasc Anesth. 2013; 27(2):e15-6.

13. Mclntosh AM, Suhong T, Deakyne SJ, Davidson JA, et al. Validation of the vasoactive inotropic score in pediatric sepsis. Pediatr Crit Care Med. 2017 Mayo. En imprenta.

14. Gordon AC, Perkins GD, Singer M, Orme RM, et al. Levosimendan for the prevention of acute organ dysfunction in sepsis. N Engl J Med. 2016;375:1638-1648.

Conflicto de intereses: Los autores reportan que no hay conflicto de intereses. No se requirió financiamiento externo.

Correspondencia:

Dr. Humberto Alfonso Castillejos Suastegui

Sur 136 Núm. 116

Col. Las Américas,

Del. Álvaro Obregón, 01120, Ciudad de México.

Tel: 52308000 , ext. 8594

Cel: 5525600337

E-mail: hacsuastegui@gmail.com 